\title{
Diagnostic Role of the Presence of Red Blood and Epithelial Cells in Nipple Discharge Cytology
}

Sundara Rajan $\mathbf{S}^{1 *}$, Verma $\mathbf{R}^{1}$, Kelly $\mathbf{J}^{2}$, Cullen $\mathbf{A}^{3}$, Turton $\mathbf{P}^{1}$, Achuthan $\mathbf{R}^{1}$ and Horgan $\mathbf{K}^{1}$

${ }^{1}$ Department of Breast Surgery, Leeds Teaching Hospitals NHS Trust, UK

${ }^{2}$ Department of Pathology, Leeds Teaching Hospitals NHS Trust, UK

${ }^{3}$ Department of Cytopathology Royal Liverpool and Broadgreen University Hospitals NHS Trust, UK

\begin{abstract}
Introduction: Management of nipple discharge (ND) is variable reflecting the lack of a uniformly accepted assessment policy. The aim of this study was to determine the common underlying pathology in patients presenting with ND as well as to evaluate the diagnostic role of red blood cells (RBC) and epithelial cells in the nipple cytology $(\mathrm{NC})$.
\end{abstract}

Methodology: Electronic data base held at Leeds Teaching Hospitals NHS Trust was searched to identify all the patients who underwent Hadfield's (open diagnostic major duct excision) procedure for ND from January 2004 to December 2009. We included only patients with ND as the primary symptom and excluded those with any underlying palpable or radiological abnormality.

Results: Hadfield's procedure was performed in 120 cases (Median age $=49$ years; range $=20-88$ ). The final histology showed benign changes (duct ectasia/hyperplasia and fibrocystic changes) in most $(37.5 \%)$, followed by intra-ductal papilloma $(32.5 \%)$, normal $(16.6 \%)$, malignant $(10.9 \%)$ and atypical $(2.5 \%)$ changes in the rest. The presence of RBC in NC was $87 \%$ sensitive to detect any underlying pathology and $69 \%$ sensitive to predict malignancy at the expense of specificity. However, epithelial cells were found to be neither sensitive nor specific enough to detect underlying pathology or malignancy.

Conclusion: The aetiology remains benign in most patients presenting with ND in the absence of any underlying palpable or radiological abnormality. Even in patients without an underlying radiological abnormality, diagnostic surgery should be offered if RBC is present in the nipple cytology.

Keywords: Nipple discharge; Nipple cytology; Breast neoplasms; Epithelial cells; Erythrocytes

\section{Introduction}

Nipple discharge (ND) is the presenting symptom in 5 to $10 \%$ of patients who attend a symptomatic breast clinic [1]. Most cases of ND are physiological in nature and are multi-ductal in origin. In these instances and particularly when associated with a creamy yellow, white or green coloured discharge, further investigations are rarely required. The term pathological nipple discharge (PND) has been introduced to describe a bloody, serous or clear discharge which is spontaneous, unilateral, uniductal and persistent [2,3]. The majority of PND is caused by benign pathology such as an intra ductal papilloma (IDP) or duct ectasia [4]. However, in up to $5 \%$ it is caused by an underlying malignancy [5]. The incidence of associated malignancy is reported to range from 9.3 to $21.3 \%$, when the ND is associated with a palpable or radiological abnormality [6].

In practice, patients with a PND are assessed clinically and radiologically. Additionally, nipple discharge cytology (NC) is widely used and is considered as a valuable investigation for patients presenting with ND. When suspicious or malignant epithelial cells are present in the $\mathrm{NC}$, it has reliable specificity and negative predictive value for detecting malignancy but at the expense of a low sensitivity $[7,8]$. Currently a review of the medical literature does not provide a predictive model for diagnosing an underlying pathology in those ND patients who present without any associated clinical or radiological abnormalities. This has resulted in many ND patients being offered a diagnostic surgical procedure (major duct excision or microdochectomy) to rule out associated malignancy [9]. Hence, the availability of any single or combination of investigative methods to identify ND patients who are at low risk of having a concurrent malignancy would be of value.
We conducted this study in a selective group of patients, who underwent diagnostic surgical excision of the major duct(s) after presenting with ND but without any underlying palpable or radiological abnormality. Our primary objective was to determine the common underlying pathology causing ND in these patients. Additionally we sought to determine the diagnostic role of red blood cells (RBC) and normal or benign epithelial cells in the NC to identify any causative pathology.

\section{Materials and Methods}

Patients who had either major duct excision or microdochectomy over a five-year period from January 2004 to December 2009 were identified from the electronic data base held at the Leeds Teaching Hospitals NHS Trust. Patient case notes were then reviewed to gather additional information when necessary. Patients were excluded, if they were found to have had any underlying palpable or radiological abnormality or if the surgery was performed for peri-ductal mastitis and sepsis.

*Corresponding authors: Sundara Rajan S, Research Fellow in Breast Surgery, Breast Secretariat, Leeds General Infirmary, Great George Street, Leeds, LS1 3EX, UK, Tel: 0044113 2265496; E-mail: s.sundara-rajan@mbht.nhs.uk

Received July 29, 2013; Accepted November 29, 2013; Published December 01, 2013

Citation: Sundara Rajan S, Verma R, Kelly J, Cullen A, Turton P, et al. (2013) Diagnostic Role of the Presence of Red Blood and Epithelial Cells in Nipple Discharge Cytology. J Cytol Histol 4: 199. doi: 10.4172/2157-7099.1000199

Copyright: (C) 2013 Sundara Rajan S, et al. This is an open-access article distributed under the terms of the Creative Commons Attribution License, which permits unrestricted use, distribution, and reproduction in any medium, provided the original author and source are credited. 
All of the patients in this series who had presented with ND underwent assessment with clinical, radiological and cytological testing. A detailed history which included the characteristics of the nipple discharge was obtained. Patients underwent either a central breast ultrasound (USS) to evaluate the retro-areolar ducts if under 35 years of age or both USS and mammogram if older than 35 years. In cases where the ND could be expressed, a smear was made on a glass slide for microscopy after being wet fixed using alcohol. The Papanicolau smear was evaluated by an experienced Cytopathologist and was classified based on the presence of RBC, epithelial cells (benign or atypical) and malignant cells. In our cohort, we did not identify any patients with atypical epithelial cells or malignant cells.

The reasons for offering diagnostic surgery varied across our retrospective group. It was primarily offered for those, where any epithelial cells were identified on NC irrespective of whether the epithelial cells were atypical in appearance or not. This was the departmental management protocol during the period of the study. Diagnostic surgery was also offered to those with spontaneous clear or blood stained ND, particularly if it was noted to be of single duct in origin. In a minority $(n=9)$, the surgery was offered for symptomatic relief due to persistent and troublesome ND. Further therapeutic resection was performed in those cases where the diagnostic surgical excision showed malignancy with incomplete excision. The histopathological specimen was examined and reported in accordance with the National Co-ordinating Group for Breast Screening Pathology Guidelines [10].

In this study for descriptive purpose, the histopathology of the excised breast tissue was defined as benign changes when duct ectasia (DE), fibrocystic changes or ductal hyperplasia without atypia were found. Intraductal papilloma (IDP) was considered as a separate entity due to being the single most common pathology causing a PND. We used a further category, benign lesion with atypia, to include, Flat Epithelial Atypia (FEA), Atypical Intraductal Proliferation (AIDP), Atypical Ductal Hyperplasia (ADH), Atypical Lobular Hyperplasia (ALH) and Lobular Carcinoma In-situ (LCIS), when present alone or in combination in the histopathology specimen in line with the National Co-ordinating Group for Breast Screening Pathology Guidelines [10]. For data interpretation, we defined the diagnostic surgical histology as having an underlying pathology, if there was IDP, benign lesion with atypia and in situ or invasive cancer. Conversely, when only benign changes or no abnormality was identified the pathology was considered to be normal. All the data were entered in to Microsoft Excel sheet and statistical analysis was performed using SPSS.v.17.0.

\section{Results}

120 open diagnostic major duct excisions (mentioned as 'cases' from now onwards for descriptive purposes) were performed during the study period on 115 patients (median age: 49 years; range: $20-88$ years). The ND was unilateral in 106 patients and bilateral in 9 patients ( 5 had bilateral and 4 unilateral major duct excisions). Ninety nine cases had either clear or blood stained ND $(82.5 \%)$. The ND was uniductal in 60 cases, multi-ductal in 14 and was not documented in 46 cases. The discharge was spontaneous in $112(93.3 \%)$ and not documented in the remaining 8 cases $(6.7 \%)$.

One hundred and eleven cases (92.5\%) underwent diagnostic surgery either due to the presence of epithelial cells on NC (which was the unit protocol over that period) or as a result of convincing spontaneous clear or blood stained ND. Diagnostic surgery due to persistent and troublesome symptomatic ND was undertaken in only 9 cases (1 case had pleomorphic lobular carcinoma in situ (PLCIS), $1 \mathrm{DE}$, 1 IDP and normal histology in the remaining).

Among our cohort, NC showed either RBC and/or epithelial cells in 99 cases $(82.5 \%)$ and did not show any abnormality in the remaining $21(17.5 \%)$. The comparison between NC and the final histological diagnosis is depicted in Table 1 The final histology showed normal or benign pathology in 65 cases (54.1\%), IDP in 39 (32.5\%), benign lesions with atypia in $3(2.5 \%)$, in situ cancer in $11(9.2 \%)$ and invasive cancer in 2 cases (1.7\%). The sensitivity of the presence of RBC in NC to predict malignancy was $69 \%$, but the specificity was only $21 \%$. Similarly, the presence of RBC had a sensitivity of $87 \%$ to detect any underlying pathology but again the specificity was only $29 \%$. The presence of epithelial cells was neither sensitive nor specific enough to detect malignancy (39\% and $51 \%$ respectively) or any underlying pathology ( $49 \%$ and $54 \%$ respectively) (Table 2 ).

In our series of 120 cases, we identified cancers in thirteen. Of these, 2 were invasive and 11 were in situ. In the two cases with invasive cancer, the initial diagnostic major duct excision histology showed ADH and in situ cancer and the invasive component was only identified on further therapeutic excision. They both underwent sentinel lymph node biopsy (SLNB) and isolated tumour cells were identified in the axillary lymph nodes of the latter patient. In the 11 cases found to have in situ cancer, no further therapeutic surgery was undertaken in 2 cases due to the complete excision of the in situ focus. The histological examination of the further therapeutic surgical excision showed residual in situ cancer in seven cases. No residual cancer was found in the remaining 2 cases .

\section{Nipple cytology}

Final histology

\begin{tabular}{|l|c|c|c|c|c|c|}
\hline & Normal (20) & Benign changes (45) & IDP (39) & Benign lesion with atypia (3) & In situ cancer (11) & Invasive cancer (2) \\
\hline No epithelial cells or RBC (21) & 7 & 8 & 3 & 0 & 0 \\
\hline Epithelial cell (5) & 1 & 3 & 0 & 1 & 0 \\
\hline RBC (42) & 8 & 12 & 16 & $1^{\dagger}$ & $1^{*}$ \\
\hline Epithelial cell+RBC (52) & 4 & 22 & 20 & 2 & 0 \\
\hline
\end{tabular}

†This patient was found to have benign lesion with atypia on diagnostic major duct excision; *this patient was found to have in situ cancer on diagnostic major duct excision

Table 1: Comparison of nipple cytology findings with that of the final histology.

\begin{tabular}{|c|c|c|c|c|c|c|c|c|}
\hline \multirow[t]{2}{*}{ Nipple cytology variables } & \multicolumn{4}{|c|}{ Any underlying pathology } & \multicolumn{4}{|c|}{ Malignancy } \\
\hline & Sensitivity & Specificity & PPV* & $\mathbf{N P V}^{\dagger}$ & Sensitivity & Specificity & PPV* & $\mathbf{N P V}^{\dagger}$ \\
\hline Presence of RBC (n=94) & 87 & 29 & 51 & 73 & 69.2 & 20.6 & 9.6 & 84.6 \\
\hline Presence of epithelial cells $(n=57)$ & 49 & 53.84 & 47.4 & 55.6 & 38.5 & 51.4 & 8.7 & 87.3 \\
\hline
\end{tabular}

${ }^{*} \mathrm{PPV}=$ Positive predictive value; ${ }^{\dagger}$ Negative predictive value

Table 2: Predictability of individual components of the nipple cytology. 


\section{Discussion}

Over the years various diagnostic methods have been proposed to investigate nipple discharge. This has included standard triple assessment, NC, ductography, ductal lavage and nipple aspiration fluid analysis $[2,8,11]$. Unfortunately none of these diagnostic modalities either alone or in combination has provided a reliable diagnostic predictive model for the management of ND in patients who present without any underlying palpable or radiological abnormality.

In comparison to other diagnostic tests, NC is widely available within the National Health Service and does have a high specificity when atypical, suspicious or malignant cells are present [12]. This means, the presence of atypical, suspicious or malignant cells in the NC correlates well with any underlying pathology and a negative test with that of a benign pathology but comes at the expense of low sensitivity $[8,12]$. The presence of underlying malignancy remains to be the main concern for the clinicians, when managing patients presenting with ND. The reported incidence of breast cancer in ND patients without any underlying palpable or radiological abnormality can be as high as $24 \%$ [13] but generally ranges from 2.5 to $6.1 \%$ [14]. In our series, cancer was diagnosed in $10.8 \%$ of the cases ; invasive cancer in 2 and in situ cancer in 11 cases.

In the current study, we evaluated the diagnostic role of epithelial cells and RBC present in the ND. It has been hypothesised that with increasing ductal epithelial abnormality, there is an increase in the number of exfoliated cells that are likely to be present in the NC [15]. Additionally, it has been suggested that women with atypical ductal epithelial cells in the $\mathrm{NC}$ are at an increased risk of developing future breast cancer [16]. It should be emphasised that, all the patients $(n=58)$ in this study found to have epithelial cells in the NC were devoid of any cytological atypia/malignancy. When the presence of any epithelial cells in the $\mathrm{NC}$ was used as a predictive test for detecting any underlying pathology, we found that the sensitivity was $49 \%$ with a specificity of $54 \%$ and a positive and negative predictive value of $47 \%$ and $56 \%$ respectively. Similar findings were shown in the only other study which evaluated the diagnostic role of epithelial cells in ND patients without any underlying palpable or radiological abnormality [14]. However, when the presence of any RBC in NC was used as a predictive test for detecting any underlying pathology, we found that the sensitivity was $87 \%$ with a specificity of $29 \%$, positive predictive value of $51 \%$ and negative predictive value of $73 \%$. In a comparable study population, our finding was supported by Chaudary et al. [17] but more recently in a cohort of 86 patients, Richards et al. [18] found that the presence of RBC does not have any predictive role. The lack of a representative population (due to inclusion of patients with palpable and/or radiological abnormalities) made it difficult to extrapolate the findings of the remaining 2 studies conducted in this field $[19,20]$.

The presence of epithelial cells in the NC was more specific in identifying any underlying pathology or malignancy. However based on our results, a PPV of around 50\% would not justify the use of epithelial cells alone in the NC to aid in the diagnostic work up of ND patients. In comparison to epithelial cells, we identified the presence of RBC in the NC as having a higher sensitivity for identifying malignancy or any underlying pathology (Table 2). Hence the false negative rates were kept to a minimum increasing the diagnostic reliability of RBC in the NC but come at the expense of a low specificity. Our results supports the potential for using Haem stick testing (Dipstick) in the assessment of $\mathrm{RBC}$ present in the ND. This would be especially useful when the colour of the ND remains uncertain for the presence of blood as in patients with dark green, brown or black ND. Dipstick has around 90\% sensitivity to detect more than $3 \mathrm{RBC}$ in a high power field, which is comparable to that of NC and also provides the results instantaneously [21].

Certain limitations of our study should be discussed as it may have influenced the generalisability of the results. The retrospective nature of the study as well as the missing data by virtue of it should have led to the occurrence of bias. The missing data also meant that the predictive role of the ND characteristics could not be evaluated in the current study. However all our patients underwent diagnostic surgical excision, we were able to correlate the $\mathrm{NC}$ findings with that of the surgical histopathology increasing the reliability of our results.

\section{Conclusion}

In most of the patients presenting with ND without any underlying palpable or radiological abnormality, the aetiology is benign. Diagnostic surgery is indicated when RBC is detected in patients presenting with a PND. The presence of epithelial cells in the NC has higher specificity compared to RBC but poor sensitivity for detecting any underlying pathology or malignancy. We would therefore advocate that, future prospective studies are needed to confirm or refute the diagnostic ability of epithelial cells as well as the role of $\mathrm{NC}$ as a diagnostic tool in patients presenting with PND.

\section{References}

1. Seltzer MH (2004) Breast complaints, biopsies, and cancer correlated with age in 10,000 consecutive new surgical referrals. Breast J 10: 111-117.

2. Sauter ER, Wagner-Mann C, Ehya H, Klein-Szanto A (2007) Biologic markers of breast cancer in nipple aspirate fluid and nipple discharge are associated with clinical findings. Cancer Detect Prev 31: 50-58.

3. Gupta RK, Gaskell D, Dowle CS, Simpson JS, King BR, et al. (2004) The role of nipple discharge cytology in the diagnosis of breast disease: a study of 1948 nipple discharge smears from 1530 patients. Cytopathology 15: 326-330.

4. Shah VI, Raju U, Chitale D, Deshpande V, Gregory N, et al. (2003) Falsenegative core needle biopsies of the breast: an analysis of clinical, radiologic, and pathologic findings in 27 concecutive cases of missed breast cancer. Cancer 97: 1824-1831.

5. Sakorafas GH (2001) Nipple discharge: current diagnostic and therapeutic approaches. Cancer Treat Rev 27: 275-282.

6. Gioffrè Florio M, Manganaro T, Pollicino A, Scarfo P, Micali B (1999) Surgical approach to nipple discharge: a ten-year experience. J Surg Oncol 71: 235-238.

7. Lau S, Küchenmeister I, Stachs A, Gerber B, Krause A, et al. (2005) Pathologic nipple discharge: surgery is imperative in postmenopausal women. Ann Surg Oncol 12: 546-551.

8. Dinkel HP, Gassel AM, Müller T, Lourens S, Rominger M, et al. (2001) Galactography and exfoliative cytology in women with abnormal nipple discharge. Obstet Gynecol 97: 625-629.

9. Dillon MF, Mohd Nazri SR, Nasir S, McDermott EW, Evoy D, et al. (2006) The role of major duct excision and microdochectomy in the detection of breast carcinoma. BMC Cancer 6: 164

10. NHSBSP (2005) Pathology Reporting of Breast Disease, 1-144

11. Van Zee KJ, Ortega Pérez G, Minnard E, Cohen MA (1998) Preoperative galactography increases the diagnostic yield of major duct excision for nipple discharge. Cancer 82: 1874-1880.

12. Cabioglu N, Hunt KK, Singletary SE, Stephens TW, Marcy S, et al. (2003) Surgical decision making and factors determining a diagnosis of breas carcinoma in women presenting with nipple discharge. J Am Coll Surg 196: 354-364.

13. Montroni I, Santini D, Zucchini G, Fiacchi M, Zanotti S, et al. (2010) Nipple discharge: is its significance as a risk factor for breast cancer fully understood? Observational study including 915 consecutive patients who underwent selective duct excision. Breast Cancer Res Treat 123: 895-900. 
Citation: Sundara Rajan S, Verma R, Kelly J, Cullen A, Turton P, et al. (2013) Diagnostic Role of the Presence of Red Blood and Epithelial Cells in Nipple Discharge Cytology. J Cytol Histol 4: 199. doi: 10.4172/2157-7099.1000199

14. Lanitis S, Filippakis G, Thomas J, Christofides T, Al Mufti R, et al. (2008) Microdochectomy for single-duct pathologic nipple discharge and normal or benign imaging and cytology. Breast 17: 309-313.

15. Dooley WC, Ljung BM, Veronesi U, Cazzaniga M, Elledge RM, et al. (2001) Ductal lavage for detection of cellular atypia in women at high risk for breast cancer. J Natl Cancer Inst 93: 1624-1632.

16. Fabian CJ, Kimler BF, Zalles CM, Klemp JR, Kamel S, et al. (2000) Short-term breast cancer prediction by random periareolar fine-needle aspiration cytology and the Gail risk model. J Natl Cancer Inst 92: 1217-1227.

17. Chaudary MA, Millis RR, Davies GC, Hayward JL (1982) The diagnostic value of testing for occult blood. Ann Surg 196: 651-655.
18. Richards T, Hunt A, Courtney S, Umeh H (2007) Nipple discharge: a sign of breast cancer? Ann R Coll Surg Engl 89: 124-126.

19. Morrogh M, Park A, Elkin EB, King TA (2010) Lessons learned from 416 cases of nipple discharge of the breast. Am J Surg 200: 73-80.

20. El-Daly H, Gudi M (2010) Evaluation of nipple discharge cytology and diagnostic value of red blood cells in cases with negative cytology: a cytohistologic correlation. Acta Cytol 54: 560-562.

21. Sokolosky MC (2001) Hematuria. Emerg Med Clin North Am 19: 621-632. 